\title{
DESEMPENHO AGRONÔMICO DE PROGÊNIES DE MARACUJAZEIRO-ROXO E MARACUJAZEIRO-AZEDO NO DISTRITO FEDERAL ${ }^{1}$
}

\author{
SIDNEY ALMEIDA FILGUEIRA DE MEDEIROS², MÁRCIO CARVALHO PIRES ${ }^{3}$, \\ OSVALDO KIYOSHI YAMANISHI ${ }^{4}$, JOSÉ RICARDO PEIXOTO ${ }^{5}$, \\ NILTON TADEU VILELA JUNQUEIRA ${ }^{6}$, JULIANA GALVARROS BUENO LOBO RIBEIRO
}

RESUMO - O maracujá-roxo é uma fruta que, em boas condições de produtividade e qualidade, pode transformar-se numa fonte de renda importante para o agricultor, devido ao elevado valor pago no mercado europeu pela fruta in natura. Com o objetivo de avaliar a produção de frutos de maracujazeiro-roxo provenientes da Austrália, em condições de cerrado, foi realizado um experimento com delineamento em blocos casualizados, com nove tratamentos, sete plantas úteis por parcela e 4 repetições. Os acessos de maracujazeiro-roxo foram: 'Lacey', '14', '25', 'Supersweet 9', '37(1)', '37(2)' e '96A'. Como testemunha, foi avaliado o acesso de maracujazeiro-azedo 'Marília Seleção Cerrado' e do híbrido 'BRS Ouro Vermelho'. Com base nos resultados, pode-se concluir que as testemunhas apresentaram as maiores produções e, dentre as variedades de maracujazeiro-roxo, o acesso '14' foi o mais produtivo, enquanto o acesso 'Lacey' foi o que apresentou o maior número de frutos colhidos, o maior peso total de frutos, o maior número de frutos por planta, frutos por hectare e frutos da classe A. A variedade 'S9' apresentou o maior percentual de frutos roxos, maior peso médio de frutos e maior percentual de frutos da classe A.

Termos para indexação: Passiflora edulis f. flavicarpa, produtividade, classificação, exportação.

\section{BEHAVIOR OF PURPLE PASSION FRUIT AND YELLOW PASSION FRUIT GENOTYPES IN THE FEDERAL DISTRICT, BRAZIL}

\begin{abstract}
The purple passion fruit with suitable yield and quality can become an important income source for growers due to high price achieved in the European fresh fruit market. With the aim to evaluate purple passion fruit varieties from Australia under Brazilian savanna conditions an experiment was carried out in a randomized blocks with 9 genotypes of passion fruit, 7 plants per plot and 4 replications. The purple passion fruit genotypes used were: 'Lacey', '14', '25', 'Supersweet 9', '37(1)', '37(2)' and '96A'. The yellow passion fruit genotypes 'Marília Seleção Cerrado' and Hybrid 'BRS Ouro Vermelho' were used as control. It can be concluded that 'BRS Ouro Vermelho' hybrid and 'Marília Seleção Cerrado' had the highest yields and among the purple passion fruit the ' 14 ' genotype showed the highest yield, while, 'Lacey' genotype had the highest total fruit weight and number of harvested fruits, fruits per plant, fruits per hectare and class "A" fruits. Genotypes 'S9' had the highest average fruit weight and percentages of purple fruits and class "A" fruits.
\end{abstract}

Index terms: Passiflora edulis Sims, P. edulis Sims f. flavicarpa Degener, yield, classification, exportation.

\section{INTRODUÇÃO}

A produtividade nacional do maracujazeiroazedo, Passiflora edulis f. flavicarpa, é relativamente baixa, entre 10 e $15 \mathrm{t} / \mathrm{ha} /$ ano, devido principalmente à baixa utilização de tecnologia de produção (Meletti \& Maia, 1999). A produtividade média, em 1996, era de $9,2 \mathrm{t} / \mathrm{ha} /$ ano e, em 2002, atingiu $13,76 \mathrm{t} / \mathrm{ha} /$ ano, porém não é raro encontrar propriedades que produzam acima de $40 \mathrm{t} / \mathrm{ha} /$ ano (Agrianual, 2005). No Distrito Federal, segundo o Agrianual (2005), a produtividade do maracujazeiro-azedo, em média, é de 15,6 t/ha/ano. Junqueira et al. (1999) afirmam que, no Distrito Federal, sob irrigação, manejo adequado e polinização, o maracujazeiro-azedo pode produzir até 70 t/ha num período de três anos, com pico de

\footnotetext{
${ }^{1}$ (Trabalho 153-08). Recebido em: 13-06-2008. Aceito para publicação em: 30-04-2009.

${ }^{2}$ Eng. Agr. MsC. Faculdade de Agronomia e Medicina Veterinária, Universidade de Brasília. sidneymedeiros@unb.br

${ }^{3}$ Eng. Agr. MsC. Faculdade de Agronomia e Medicina Veterinária, Universidade de Brasília. mcpires@unb.br

${ }^{4}$ Eng. Agr. Dr. Faculdade de Agronomia e Medicina Veterinária, Universidade de Brasília. kiyoshi@unb.br

${ }^{5}$ Eng. Agr. Dr. Faculdade de Agronomia e Medicina Veterinária, Universidade de Brasília. peixoto@unb.br

${ }^{6}$ Eng. Agr. Dr. Embrapa Cerrados - Brasília/DF. junqueir@cpac.embrapa.br

${ }^{7}$ Eng. Agr. Faculdade de Agronomia e Medicina Veterinária, Universidade de Brasília. julianagalvarros@brturbo.com.br
} 
produção, no segundo ano, de até 40 t/ha.

A produtividade de maracujazeiro-roxo, no Brasil é baixa, em torno de $5 \mathrm{t} / \mathrm{ha} / \mathrm{ano}$. Entretanto, isto não representa uma limitação a seu cultivo, pois para o maracujá-roxo, a qualidade dos frutos é mais importante que a produção. Apesar de seus frutos serem bastante alternantes quanto ao formato, há que se considerar conjuntamente a produção com as demais características. O fruto do maracujazeiroroxo Australiano representa a preferência do mercado internacional, pelo seu elevado teor de sólidos solúveis e baixa acidez, que dispensa o uso de açúcar quando consumido e eleva o rendimento industrial na agroindústria (Toda Fruta, 2002).

Segundo São José (1994), a colheita do maracujazeiro-azedo, no Brasil, inicia-se a partir do quinto até o décimo mês após o plantio, e a produção é de seis a doze meses por ano, dependendo das condições ambientais e da localização geográfica. O período de colheita que concentra a maior quantidade de frutos vai de janeiro a março (Ruggiero, 1987). Já produção de maracujazeiro-roxo, na Austrália, ocorre durante todo o ano, com um pico em dezembro e janeiro, e outros dois picos menores em março/abril e julho/agosto (Newett et al., 1998).

O maracujá-roxo pode ser colhido na planta e completar seu amadurecimento fora dela, seja com a indução por etileno, ou não (Salomão et al., 2001). Arjona \& Matta (1991) colheram frutos de casca verde fisiologicamente maduros, entre 55 e 60 dias após a antese, e após tratamento com etileno, os mesmos desenvolveram a coloração roxa normal e os mesmos níveis de sólidos solúveis e $\mathrm{pH}$ que os frutos colhidos sobre o solo após a abscisão da planta. Aular et al. (1995), analisando frutos de maracujazeiro-azedo, também observaram que frutos colhidos diretamente das plantas, após a maturação fisiológica, apresentaram características físico-químicas semelhantes aos colhidos sobre o solo.

Segundo levantamentos de Durigan et al. (2004), não existem normas ou regulamentos técnicos oficiais para a identidade e a qualidade de maracujás, apenas padrões relacionados ao diâmetro, peso, cor, textura, teor de sólidos solúveis e acidez. Oliveira (2001), avaliando produtividade e severidade de doenças em acessos de maracujazeiro-azedo cultivados sob adubação potássica, utilizou a classificação da Tabela 1. De acordo com padrões internacionais, o maracujá-roxo classifica-se conforme mostra a Tabela 2.

O presente trabalho teve como objetivo avaliar agronomicamente sete acessos de maracujazeiroroxo provenientes da Austrália e dois acessos de maracujazeiro-azedo utilizados comercialmente no
Brasil, através da quantificação das produtividades total e estratificada em diferentes classes de frutos.

\section{MATERIAL E MÉTODOS}

O experimento foi realizado de fevereiro de 2004 a abril de 2005, no Setor de Fruticultura da Estação Experimental da Biologia - EEB, Universidade de Brasília, situada no Distrito Federal, a uma latitude sul de $16^{\circ}$, longitude a oeste de Greenwich de $48^{\circ}$, e altitude de $1.010 \mathrm{~m}$ acima do nível do mar. O clima da região é do tipo $\mathrm{AW}$, caracterizado por chuvas concentradas no verão, de outubro a abril, e invernos secos, de maio a setembro (Melo, 1999). O delineamento experimental utilizado foi o de blocos casualizados, com nove tratamentos, sete plantas úteis por parcela e quatro repetições, numa área total de 0,25 hectare.

Os acessos testados foram: 'Marília Seleção Cerrado' ('MSC'); 'Lacey'; '14'; '25'; 'Supersweet 9' ('S9'); '37(1)' - 'Misty Gem 37' ou 'Supersweet 7'; '37(2)' - 'Misty Gem 37' ou 'Supersweet 7'; '96A' ou 'Supersweet 4' e o híbrido 'BRS Ouro Vermelho'. O acesso 'Marília Seleção Cerrado' e híbrido 'BRS Ouro Vermelho' são produtores de maracujás- amarelos ou róseos, mas foram utilizados no experimento como testemunhas, já que o primeiro é cultivado em larga escala na região Centro-Oeste do Brasil, e o segundo foi recentemente lançado como híbrido comercial.

Os nove acessos foram semeados em tubetes plásticos de $300 \mathrm{~mL}$, preenchidos com substrato artificial, à base de vermiculita e casca de Pinus sp., cultivados sob estufa. Utilizaram-se seis gramas de adubo NPK Osmocote ${ }^{\circledR}$ (14-14-14) por litro de substrato.

O experimento foi instalado em um solo tipo Latossolo Vermelho, fase argilosa profundo, com boa drenagem e baixa fertilidade natural, apresentando as seguintes características: $\mathrm{Al} 0,1 \mathrm{mE} / 100 \mathrm{~mL} ; \mathrm{Ca}+\mathrm{Mg}$ 1,75 mE/100mL; P 5 mg.L $L^{-1}$; K 0,3 mE/100mL; pH 5,75; saturação de Al 5,5\%; saturação de bases 33 $\%$; e matéria orgânica 2,49 \%. Foram aplicados 200 $\mathrm{g}$ de calcário dolomítico no fundo de cada cova, cujas dimensões eram de 40 x 40 x $40 \mathrm{~cm}$. Além disso, foram acrescentados $10 \mathrm{~kg}$ de esterco curtido de ovino, $0,5 \mathrm{~kg}$ de Yoorin Master ${ }^{\circledR}$ e $0,5 \mathrm{~kg}$ de superfosfato simples por cova, incorporados ao solo.

As mudas foram transplantadas para o campo, 105 dias após o semeio, no espaçamento de $2,5 \mathrm{~m}$ entre linhas e entre plantas, totalizando 1.600 plantas por hectare. As adubações de cobertura foram realizadas quinzenalmente. As plantas foram conduzidas em espaldeira vertical, com os mourões distanciados 
de $7 \mathrm{~m}$ e um fio de arame liso a $2 \mathrm{~m}$ de altura em relação ao solo. As plantas foram conduzidas em haste única, tutoradas por barbante, até o fio de arame, deixando-se duas brotações laterais em sentido oposto, ao longo do arame. Foi utilizada irrigação suplementar desde o plantio no campo até o início das chuvas, em novembro de 2004. Foram realizadas pulverizações com fungicida sistêmico alternado com protetor, em intervalos de 15 dias, durante o período de crescimento vegetativo até a primeira colheita, para o controle de doenças.

Não se realizou polinização artificial para aumentar a frutificação. As colheitas foram realizadas uma vez por semana, recolhendo somente os frutos que se encontravam no solo. Os frutos colhidos foram contados e classificados por cor e tamanho e depois pesados. Para a classificação do maracujá-amarelo, foi utilizada a tabela de Oliveira (2001) (Tabela 1); já a classificação dos acessos de maracujazeiro-roxo foi feita a partir da tabela de Durigan et al. (2004) (Tabela 2). Esta divisão na classificação foi necessária devido às diferenças entre os frutos produzidos pelos dois tipos de maracujazeiro.

A colheita de frutos para análises foi realizada no período de 27 de janeiro a 26 de abril de 2005 , totalizando 13 colheitas. As variáveis quantificadas foram: número de frutos de cor amarela, rosa e roxa; número total e peso total de frutos; peso médio dos frutos; número médio de frutos por planta; número total de frutos por hectare, produtividade em $\mathrm{kg} / \mathrm{ha}$; número total de frutos de cada classe, peso total dos frutos de cada classe, e peso médio dos frutos de cada classe.

$\mathrm{Na}$ análise de variância dos dados, para avaliação da significância do efeito dos tratamentos, foi utilizado o teste de F. Compararam-se as medidas entre si, pelo teste de Tukey, ao nível de 5\% de probabilidade (Banzatto \& Kronka, 1992).

As análises estatísticas foram executadas, utilizando-se do software SANEST (Zonta e Machado, 1995).

\section{RESULTADOS E DISCUSSÃO}

Os dois acessos de maracujazeiro-azedo 'BRS Ouro Vermelho' e 'Marília Seleção Cerrado' - apresentaram, significativamente, os frutos mais pesados entre todos os genótipos avaliados. $\mathrm{O}$ acesso de maracujazeiro-roxo que apresentou o maior peso médio dos frutos, foi o 'S9'. O acesso 'Lacey' produziu o maior número total de frutos. Entre os acessos de maracujá-roxo, 'Lacey' também foi o que apresentou o maior peso total de frutos. No geral, 'Lacey' produziu o maior número de frutos por planta e, consequentemente, o maior número de frutos por hectare, porém sem diferir significativamente dos demais. $\mathrm{O}$ acesso '14' foi o que apresentou a maior produtividade por hectare entre os acessos de maracujazeiro-roxo, porém não houve diferença significativa. Já entre os acessos de maracujazeiroazedo, o híbrido 'BRS Ouro Vermelho' foi o mais produtivo, diferindo significativamente de todos os acessos de maracujazeiro-roxo (Tabela 3 ). O acesso 'S9' apresentou o maior percentual de frutos de cor roxa $(99,3 \%)$ e 'MSC' apresentou $100 \%$ de frutos amarelos (Figura 1).

A produtividade registrada nos dois tipos de maracujazeiro confirma o exposto por Manica (1981), que afirma que o maracujazeiro-azedo é mais produtivo que o maracujazeiro-roxo. Apesar de a produtividade do maracujá-roxo ser menor, este consegue no mercado valores superiores, pois a sua qualidade e destino para consumo in natura são mais importantes do que a quantidade produzida (Toda Fruta, 2002). Ainda assim, esses valores excedem a média nacional de produtividade, porque em apenas 13 colheitas ou três meses a produtividade de maracujá-amarelo foi maior do que a média anual de produção (13,76 t/ha/ano) (Agrianual, 2005), apesar de a colheita poder ser realizada durante 6 a 12 meses, dependendo da localização geográfica do pomar (São José, 1994). Se a colheita de maracujá-roxo fosse estendida por todo o ano, conforme afirmam Newett et al. (1998), dependendo do fotoperíodo da região, conseguiria alcançar a produtividade nacional de maracujá em menos de seis meses. Entretanto, a produtividade média de maracujazeiro-roxo na Austrália gira em torno de $15 \mathrm{t} / \mathrm{ha} / \mathrm{ano}$, podendo atingir até 25 t/ha/ano (Rigden, 2005). Todos os acessos de maracujazeiro-roxo, inclusive, superaram as expectativas de produtividade, visto que, de acordo com reuniões informais junto a produtores de maracujá, estima-se que as variedades de maracujazeiro-roxo existentes no Brasil produzam em torno de $5 \mathrm{t} / \mathrm{ha} /$ ano.

Na classificação do maracujazeiro-azedo, o acesso 'MSC' apresentou maior número de frutos do que o 'BRS Ouro Vermelho' nas classes 3A, 2A e $1 \mathrm{~A}$, porém não houve diferença significativa. $\mathrm{Na}$ classe Primeira, 'BRS Ouro Vermelho' obteve maior número de frutos, também sem diferir significativamente. O peso médio dos frutos foi maior em todas as classes para frutos do híbrido 'BRS Ouro Vermelho', havendo diferença significativa nas classes $2 \mathrm{~A}$ e 1A. O acesso 'MSC' apresentou maior peso total de frutos de classe 3A, e no 'BRS Ouro Vermelho' o maior peso total foi encontrado nas classes $2 \mathrm{~A}, 1 \mathrm{~A}$, e Primeira, porém não houve diferença significativa 
entre as classes (Tabela 3 ).

Na classificação do maracujazeiro-roxo, o acesso 'Lacey' apresentou o maior número de frutos em todas as classes. Na classe A, foram obtidos 507,3 frutos de 'Lacey', mas não houve diferença significativa entre os acessos de maracujazeiro-roxo. O acesso 'S9' apresentou o menor número de frutos nas classes B, C e D. Na classe B, 'S9' diferenciou-se significativamente de 'Lacey' e '37(2)'. Nas classes $\mathrm{C}$ e $\mathrm{D}$, houve diferença significativa de 'S9' apenas com 'Lacey' (Tabela 4). O maior peso médio foi obtido por 'S9' na classe A. Na classe A, apenas o acesso ' 14 ' não diferiu significativamente de 'S9'. Mesmo tendo apresentado o maior número de frutos na classe A, o acesso 'Lacey' foi o que apresentou menor peso médio de frutos nesta classe. Nas classes $\mathrm{B}, \mathrm{C}$ e D, os acessos não diferiram significativamente entre si para a variável peso médio (Tabela 6). Com relação ao peso total de frutos, o acesso 'S9' obteve o melhor resultado na classe A, porém sem diferir significativamente dos demais. Nas demais classes, o acesso 'Lacey' registrou maior peso total de frutos: na classe B, diferiu significativamente de ' 14 ', ' 25 ' e 'S9'; nas classes C e D, diferiu apenas de 'S9' (Tabela 5).

TABELA 1 - Classificação do fruto de maracujazeiro-azedo de acordo com o seu diâmetro equatorial (mm). Brasília-DF, EEB-UnB, 2005.

\begin{tabular}{cc}
\hline Classificação & Diâmetro Equatorial $(\mathbf{m m})$ \\
\hline Primeira & Igual ou menor que 65 \\
1A & Maior que 65 até 75 \\
2A & Maior que 75 até 85 \\
3A & Maior que 85 \\
\hline Fonte: Adaptada de Oliverra (2001).
\end{tabular}

TABELA 2 - Classificação do fruto de maracujazeiro-roxo de acordo com o seu peso (g) e diâmetro equatorial (mm). Brasília-DF, EEB-UnB, 2005.

\begin{tabular}{cccc}
\hline Classe & Nome & Peso $(\mathbf{g})$ & Diâmetro Equatorial $(\mathbf{m m})$ \\
\hline A & Superior & $>35$ & $47-51$ \\
B & Escolhido & $25-35$ & $44-47$ \\
C & Standard & $20-25$ & $40-44$ \\
D & Substandard & $<20$ & $38-40$
\end{tabular}

Fonte: Adaptada de Durigan et al. (2004).

TABELA 3 - Número de frutos roxos (NFR), número de frutos amarelos (NFA), número total de frutos $(\mathrm{NTF})$, peso total $(\mathrm{PT})$, peso médio $(\mathrm{PM})$, número de frutos por planta $(\mathrm{F} / \mathrm{PL})$, número de frutos por hectare (F/ha) e produtividade (Prod) de dois acessos de maracujazeiro-azedo e sete acessos de maracujazeiro-roxo. Brasília-DF, EEB-UnB, 2005.

\begin{tabular}{ccccccccc}
\hline Acessos & NFR & NFA & NTF & PT (kg) & PM (g) & F/PL & F/ha & Prod (t/ha) \\
\hline 'BRS Ouro & $78 \mathrm{~cd} *$ & $341 \mathrm{ab}$ & $419 \mathrm{a}$ & $79,7 \mathrm{a}$ & $196,0 \mathrm{a}$ & $59,9 \mathrm{a}$ & $95.886 \mathrm{a}$ & $18,2 \mathrm{a}$ \\
Vermelho' & 0d & $398 \mathrm{a}$ & $398 \mathrm{a}$ & $71,6 \mathrm{a}$ & $183,8 \mathrm{a}$ & $56,8 \mathrm{a}$ & $90.857 \mathrm{a}$ & $16,4 \mathrm{ab}$ \\
MSC & $682 \mathrm{a}$ & $20 \mathrm{c}$ & $702 \mathrm{a}$ & $33,0 \mathrm{~b}$ & $46,4 \mathrm{~b}$ & $107,1 \mathrm{a}$ & $171.285 \mathrm{a}$ & $8,0 \mathrm{c}$ \\
Lacey & $491 \mathrm{abc}$ & $49 \mathrm{bc}$ & $540 \mathrm{a}$ & $31,8 \mathrm{~b}$ & $59,1 \mathrm{~b}$ & $93,1 \mathrm{a}$ & $148.971 \mathrm{a}$ & $8,7 \mathrm{bc}$ \\
14 & $423 \mathrm{abc}$ & $63 \mathrm{bc}$ & $486 \mathrm{a}$ & $27,0 \mathrm{~b}$ & $55,1 \mathrm{~b}$ & $82,7 \mathrm{a}$ & $132.297 \mathrm{a}$ & $7,3 \mathrm{c}$ \\
25 & $480 \mathrm{abc}$ & $3 \mathrm{c}$ & $483 \mathrm{a}$ & $32,7 \mathrm{~b}$ & $67,7 \mathrm{~b}$ & $73,4 \mathrm{a}$ & $117.371 \mathrm{a}$ & $7,9 \mathrm{c}$ \\
S9 & $483 \mathrm{abc}$ & $107 \mathrm{abc}$ & $590 \mathrm{a}$ & $31,1 \mathrm{~b}$ & $53,6 \mathrm{~b}$ & $84,3 \mathrm{a}$ & $134.800 \mathrm{a}$ & $7,1 \mathrm{c}$ \\
$37(1)$ & $525 \mathrm{ab}$ & $117 \mathrm{abc}$ & $642 \mathrm{a}$ & $32,5 \mathrm{~b}$ & $50,3 \mathrm{~b}$ & $97,0 \mathrm{a}$ & $155.200 \mathrm{a}$ & $7,9 \mathrm{c}$ \\
$37(2)$ & $199 \mathrm{bcd}$ & $386 \mathrm{a}$ & $585 \mathrm{a}$ & $32,0 \mathrm{~b}$ & $54,3 \mathrm{~b}$ & $86,5 \mathrm{a}$ & $138.467 \mathrm{a}$ & $7,5 \mathrm{c}$ \\
\hline $96 \mathrm{~A}$ & 46,1 & 76,2 & 28,2 & 36,8 & 11,9 & 28,0 & 29,0 & 35,1 \\
\hline CV (\%) & & & & & & & &
\end{tabular}

Médias seguidas de mesma letra nas colunas não diferem significativamente entre si a $5 \%$ pelo teste de Tukey.

*Frutos de cor rosa. 
TABELA 4 - Número de frutos A (NFA), B (NFB), C (NFC) e D (NFD) de sete acessos de maracujazeiroroxo. Brasília-DF, EEB-UnB, 2005.

\begin{tabular}{ccccc}
\hline Acessos & NFA & NFB & NFC & NFD \\
\hline Lacey & $507,3 \mathrm{a}$ & $88,0 \mathrm{a}$ & $45,3 \mathrm{a}$ & $15,8 \mathrm{a}$ \\
14 & $451,3 \mathrm{a}$ & $26,0 \mathrm{bc}$ & $14,5 \mathrm{ab}$ & $2,0 \mathrm{~b}$ \\
25 & $403,0 \mathrm{a}$ & $25,0 \mathrm{bc}$ & $12,0 \mathrm{ab}$ & $2,8 \mathrm{ab}$ \\
$\mathrm{S} 9$ & $435,0 \mathrm{a}$ & $5,8 \mathrm{c}$ & $2,5 \mathrm{~b}$ & $1,0 \mathrm{~b}$ \\
$37(1)$ & $463,3 \mathrm{a}$ & $49,8 \mathrm{abc}$ & $29,0 \mathrm{ab}$ & $11,3 \mathrm{ab}$ \\
$37(2)$ & $467,0 \mathrm{a}$ & $68,8 \mathrm{ab}$ & $28,0 \mathrm{ab}$ & $12,3 \mathrm{ab}$ \\
$96 \mathrm{~A}$ & $457,3 \mathrm{a}$ & $51,3 \mathrm{abc}$ & $30,8 \mathrm{ab}$ & $11,5 \mathrm{ab}$ \\
\hline CV $(\%)$ & 33,0 & 47,0 & 63,0 & 71,0
\end{tabular}

Médias seguidas de mesma letra nas colunas não diferem significativamente entre si, a $5 \%$, pelo teste de Tukey.

TABELA 5 - Peso total de frutos A (PFA), B (PFB), C (PFC) e D (PFD) de sete acessos de maracujazeiroroxo. Brasília-DF, EEB-UnB, 2005.

\begin{tabular}{ccccc}
\hline Acessos & PFA(g) & PFB(g) & PFC(g) & PFD(g) \\
\hline Lacey & $26.396,3 \mathrm{a}$ & $3.101,3 \mathrm{a}$ & $1.256,3^{\mathrm{a}}$ & $320,0 \mathrm{a}$ \\
14 & $27.802,5 \mathrm{a}$ & $957,5 \mathrm{bc}$ & $435,0 \mathrm{ab}$ & $47,5 \mathrm{ab}$ \\
25 & $23.118,8 \mathrm{a}$ & $896,3 \mathrm{bc}$ & $336,3 \mathrm{ab}$ & $67,5 \mathrm{ab}$ \\
S9 & $29.875,0 \mathrm{a}$ & $202,5 \mathrm{c}$ & $65,0 \mathrm{~b}$ & $25,0 \mathrm{~b}$ \\
$37(1)$ & $25.862,5 \mathrm{a}$ & $1.763,8 \mathrm{abc}$ & $851,3 \mathrm{ab}$ & $234,5 \mathrm{ab}$ \\
$37(2)$ & $25.630,0 \mathrm{a}$ & $2.512,5 \mathrm{ab}$ & $820,0 \mathrm{ab}$ & $268,8 \mathrm{ab}$ \\
$96 \mathrm{~A}$ & $26.523,8 \mathrm{a}$ & $1.793,8 \mathrm{abc}$ & $820,0 \mathrm{ab}$ & $228,8 \mathrm{ab}$ \\
\hline CV $(\%)$ & 34,4 & 48,6 & 68,9 & 71,2 \\
\hline
\end{tabular}

Médias seguidas de mesma letra nas colunas não diferem significativamente entre si, a $5 \%$, pelo teste de Tukey.

TABELA 6 - Peso médio de frutos A (PMA), B (PMB), C (PMC) e D (PMD) de sete acessos de maracujazeiro-roxo. Brasília-DF, EEB-UnB, 2005.

\begin{tabular}{ccccc}
\hline Acessos & PMA(g) & PMB(g) & PMC(g) & PMD(g) \\
\hline Lacey & $51,8 \mathrm{c}$ & $35,6 \mathrm{a}$ & $27,6 \mathrm{a}$ & $20,3 \mathrm{a}$ \\
14 & $61,7 \mathrm{ab}$ & $36,8 \mathrm{a}$ & $29,3 \mathrm{a}$ & $18,1 \mathrm{a}$ \\
25 & $57,1 \mathrm{bc}$ & $36,2 \mathrm{a}$ & $28,1 \mathrm{a}$ & $24,2 \mathrm{a}$ \\
S9 & $68,6 \mathrm{a}$ & $36,1 \mathrm{a}$ & $26,7 \mathrm{a}$ & $25,0 \mathrm{a}$ \\
$37(1)$ & $56,5 \mathrm{bc}$ & $34,8 \mathrm{a}$ & $28,8 \mathrm{a}$ & $21,0 \mathrm{a}$ \\
$37(2)$ & $55,0 \mathrm{bc}$ & $35,9 \mathrm{a}$ & $28,8 \mathrm{a}$ & $23,7 \mathrm{a}$ \\
$96 \mathrm{~A}$ & $57,8 \mathrm{bc}$ & $34,8 \mathrm{a}$ & $26,4 \mathrm{a}$ & $19,5 \mathrm{a}$ \\
\hline CV $(\%)$ & 6,2 & 6,9 & 9,1 & 16,1
\end{tabular}

Médias seguidas de mesma letra nas colunas não diferem significativamente entre si, a $5 \%$, pelo teste de Tukey. 


\section{CONCLUSÃO}

Todos os acessos de maracujazeiro-roxo avaliados têm potencial para produção no cerrado do Distrito Federal. Os acessos de maracujazeiroazedo 'Marília Seleção Cerrado' e híbrido 'BRS Ouro Vermelho' produziram o dobro dos acessos de maracujazeiro-roxo, sendo que o 'BRS Ouro Vermelho' foi o que apresentou maior produtividade e peso médio dos frutos. Os acessos 'S9' e 'Lacey' produziram os maiores percentuais de frutos roxos e o acesso 'Lacey' produziu o maior número de frutos por planta entre todos os genótipos avaliados. Entre os acessos de maracujazeiro-roxo, a variedade ' $\mathrm{S} 9$ ' produziu os maiores frutos, enquanto a variedade ' 14 ' apresentou a maior produtividade, compensando o fato de não produzir frutos tão pesados quanto a variedade ' $\mathrm{S} 9$ '.

\section{REFERÊNCIAS}

AGRIANUAL 2004: anuário agrícola brasileiro. São Paulo: FNP Consultoria \& Agroinformativos, 2005. p.394-399.

ARJONA, H. E.; MATTA, F. B. Post harvest quality of passion fruit as influenced by harvest time and ethylene treatment. Hortscience, Alexandria, v.26, n.10, p. 1297-1298, 1991..

AULAR, J.; BAUTISTA, D.; MACIEL, N. Características físicas del fruto e químicas de la pulpa $\mathrm{Y}$ el jugo de la parchita según el estado de coloración. Bioagro, Venezuela, v.7, n.1, p.17-21, 1995.

BANZATTO, D. A.; KRONKA, S. do N. Experimentação agrícola. 2. ed. Jaboticabal: Funep, 1992. $247 \mathrm{p}$.

DURIGAN, J. F.; SIGRIST, J. M. M.; ALVES, R. E.; FILGUEIRAS, H. A. C.; VIEIRA, G. Qualidade e tecnologia pós-colheita do maracujá. In: LIMA, A. DE A.; CUNHA, M. A. P. da Maracujá: produção e qualidade na passicultura. Cruz das Almas: Embrapa Mandioca e Fruticultura Tropical, 2004. 396 p.

JUNQUEIRA, N. T. V.; ICUMA, I. M.; VERAS, M. C. M.; OLIVEIRA, M. A. S.; DOS ANJOS, J. R. N. In: SILVA, J.M. de M. (Ed.). Incentivo à fruticultura no Distrito Federal: manual de fruticultura. Brasília: COOLABORA, 1999. p.42-52.

MANICA, I. Fruticultura tropical 1: maracujá. São Paulo: Editora Agronômica Ceres, 1981. 151 p.
MELETTI, L. M. M.; MAIA, M. L. Maracujá: produção e comercialização. Campinas: Instituto Agronômico, 1999. 62 p. (Boletim Técnico, 181).

MELO, K. T. Comportamento de seis cultivares de maracujazeiro-azedo (Passiflora edulis Sims e Passiflora edulis Sims f. flavicarpa Deg.) em Vargem Bonita, no Distrito Federal. 1999. 99 f. Dissertação (Mestrado) - Universidade de Brasília, Brasília, 1999.

NEWETT, S.; DIROU, J.; VOCK, N.; RIGDEN, P. Passionfruit information kit. Queensland: Department of Primary Industries, 1998. 32 p.

OLIVEIRA, A. T. Produtividade e avaliação da incidência e severidade de doenças em frutos de nove genótipos de maracujazeiro-azedo cultivados sob influência de adubação potássica no Distrito Federal. 2001. 83 f. Dissertação (Mestrado) - Universidade de Brasília, Brasília, 2001.

RIGDEN, P. Is passion fruit growing for you? Queensland: Department of Primary Industries and Fisheries. Disponível em: $<$ http://www.dpi.qld.gov. $\mathrm{au} /$ horticulture/16828.html $>$. Acesso em: 28 jan. 2005.

RUGGiero, C. Colheita. In: RUGGIERO, C. Cultura do maracujazeiro. Ribeirão Preto: Legis Summa, 1987. p.169-172.

SALOMÃO, L. C. C.; VIEIRA, G.; MOTA, W. F. da. Tecnologia de colheita e pós-colheita. In: BRUCKNER, C.H.; PICANÇO, M.C. Maracujá: tecnologia de produção, pós-colheita, agroindústria, mercado. Porto Alegre: Cinco Continentes, 2001. 472 p.

SÃO JOSÉ, A. R. A cultura do maracujazeiro: produção e mercado. Vitória da Conquista: DFZ/ UESB, 1994. 29p. Disponível em: <www.todafruta. com.br>.

TODA FRUTA. Boletim eletrônico. Jaboticabal: Sociedade Brasileira Fruticultura, 2002. Disponível em: <www.todafruta.com.br>. Acesso em: 28 fev. 2002.

ZONTA, E. P.; MACHADO, A. A. SANEST: sistema de análise estatística para microcomputadores. Pelotas: UFPel, 1995. 48 p. (SEI n. 066060, Categoria A) 